

\title{
ANALYTIC SEMIGROUPS: APPLICATIONS TO INVERSE PROBLEMS FOR FLEXIBLE STRUCTURES ${ }^{1}$
}

\author{
H. T. Banks \\ Center for Applied Mathematical Sciences \\ University of Southern California \\ Los Angeles, CA 90089-1113 \\ and \\ D. A. Rebnord \\ Department of Mathematics \\ Iowa State University \\ Ames, IA 50011
}

\begin{abstract}
We present new convergence and stability results for least squares inverse problems involving systems described by analytic semigroups. The practical importance of these results is demonstrated by application to several examples from problems of estimation of material parameters in flexible structures using accelerometer data.
\end{abstract}

\footnotetext{
${ }^{1}$ This research was supported in part by the Air Force Office of Scientific Research under Contract F4962086-C-0111, by the National Aeronautics and Space Administration under NASA Grant NAG-1-517, and by the National Science Foundation under NSF Grant DMS-8818530. Part of this research was carried out while the first author was a visiting scientist at the Institute for Computer Applications in Science and Engineering (ICASE), NASA Langley Research Center, Hampton, VA 23665, which is operated under NASA Contract NAS1-18605.
} 
$=$ 


\section{Introduction}

In this presentation we consider abstract inverse problems in a least squares formulation for parameter dependent partial differential equations. We are interested in approximation ideas which lead to viable computational techniques for such problems. We pursue our investigations in the context of the general framework for convergence and stability developed by Banks and Ito in [BI]. Motivated by questions related to the use of accelerometer data to estimate parameters in flexible structures, we focus on second order (in time) systems with sufficient damping so that the system can be modeled by an analytic semigroup.

We state and prove a new approximation result (a Trotter-Kato type theorem) for analytic semigroups. This theorem gives conditions under which a family of approximating semigroups and all its time derivatives converges to a limit semigroup and all its time derivatives, respectively. These theoretical results are then stated in terms of simple, readily checked conditions on the sesquilinear forms defining "stiffness" and "damping" in the abstract second order systems.

We discuss several examples which indicate clearly the practical importance of these new convergence results. In two of the examples presented (the damped, cantilevered EulerBernoulli beam and the "RPL experiment" structure), we can apply the abstract theorem to substantially sharpen results already found in the research literature. In a third example involving a two dimensional grid structure, we note that the theory can be applied to obtain new results for acceleration data convergence in the least squares inverse problems.

\section{Abstract Inverse Problems}

In this section we formulate a class of inverse problems as abstract least squares optimization problems constrained by evolution equations in a Hilbert space and summarize some of our previous results for such problems $[\mathrm{B}],[\mathrm{BI}],[\mathrm{BK}]$.

We assume we are given a parameter dependent system

$$
\begin{aligned}
& \dot{u}(t)=A(q) u(t)+F(t, q), \quad 0<t<T, \\
& u(0)=u_{0}(q)
\end{aligned}
$$

for states $u(t ; q)$ in a Hilbert space $H$. The parameters $q$ are to be chosen from an admissible parameter set $Q$ contained in a metric space $\left(Q_{1}, d\right)$. We assume throughout that $Q$ is a compact subset of $Q_{1}$ (a "regularization" assumption).

We are given observations $\left\{z_{i}\right\}$, a set of points in the observation space $Z$, along with an observation map $\mathcal{C}: C(0, T ; H) \rightarrow Z$ from the states to the observations. The points $z_{i}$ are observations for $\mathcal{C} u\left(t_{i} ; q\right), 0<t_{i}<T$, and it is this "data" to which we wish to fit the model 
by a best choice of the parameter $q$. Formally, the problem can be stated as

$$
\begin{aligned}
& \text { Find } \bar{q} \in Q \text { to minimize over } Q \text { the } \\
& \text { functional } J(q)=\sum_{i}\left|\mathcal{C} u\left(t_{i} ; q\right)-z_{i}\right|_{z}
\end{aligned}
$$

The observation operator $\mathcal{C}$ is of fundamental importance to the discussions in this paper. For parabolic systems (2.1), a typical example for $\mathcal{C}$ arises from pointwise evaluation in the spatial variables, e.g., $\mathcal{C} u(t ; q)=\left\{u\left(t, x_{j} ; q\right)\right\}_{j=1}^{\ell}$ where $Z=R^{\ell}$. For problens involving structures such as beams or plates, several examples arise in practice. If one takes measurements with a laser vibrometer, then one obtains measurements at specific points in space for the velocities $u_{t}\left(t_{i}, x_{j} ; q\right)$ so that the map $\mathcal{C}$ is a composite of time differentiation $\frac{\partial}{\partial t}$ followed by pointwise evaluation. If the measuring devices are accelerometers, one has observations for the accelerations $u_{t t}\left(t_{i}, x_{j} ; q\right)$ and thus $\mathcal{C}$ is time differentiation (twice) $\frac{\partial^{2}}{\partial t^{2}}$ followed by pointwise evaluation.

The inverse problems outlined here are generally infinite dimensional in both the states $u(t) \in H$ and the parameters $q \in Q$ and, moreover, involve unbounded operators related to the states $(A(q))$ and the observations $(\mathcal{C})$. Thus to develop efficient computational methods, one must make finite dimensional approximations for both the state and parameter spaces, $H$ and $Q$, respectively. For the discussions of this paper, we shall restrict our considerations to approximations of the state space. In some cases (where the parameter sets $Q$ are finite dimensional, either naturally or through some a priori parameterization) no approximation of the parameter set is necessary. Methods for approximation of $Q$ by $\operatorname{sets} Q^{M}$ have been fully discussed elsewhere (see [BK]) and these ideas could readily be incorporated in our presentation. Since this would add nothing to either the difficulties or their resolution that are the focus here, to avoid considerable notational tedium we do not consider such approximation ideas in the discussions below.

Thus, we consider approximate (to be made precise below) state spaces $H^{N} \subset H$ with associated approximate states $u^{N}(t ; q) \in H^{N}$ satisfying the approximate systems

$$
\begin{aligned}
& \dot{u}^{N}(t)=A^{N}(q) u^{N}(t)+P^{N} F(t, q) \\
& u^{N}(0)=P^{N} u_{0}(q)
\end{aligned}
$$

where $A^{N}$ is an approximation to $A$ and $P^{N}$ is the orthogonal projection of $H$ onto $H^{N}$. The corresponding approximate inverse problems are given by

$$
\begin{aligned}
& \text { Find } \bar{q}^{N} \in Q \text { to minimize over } Q \text { the } \\
& \text { functional } J^{N}(q)=\sum_{j}\left|\mathcal{C} u^{N}\left(t_{i} ; q\right)-z_{i}\right|_{z} .
\end{aligned}
$$

Of course, there are a number of reasonable ways in which the approximations may be made to arrive at the problems $\left(\mathcal{P}^{N}\right)$. In analyzing different methods and their behavior in the context of inverse problems, a number of questions related to parameter convergence 
arise naturally. For example, given a fixed set of data $\tilde{z}=\left\{z_{i}\right\}$, do optimal parameters $\bar{q}^{N}$ of $\left(\mathcal{P}^{N}\right)$ converge in some sense to an optimal parameter $\bar{q}$ for $(\mathcal{P})$ ? More generally, one might also incorporate the continuous dependence of the estimates on the observations in the concept of method stability [B], [BK]. For this, one denotes by $\bar{q}^{N}\left(\tilde{z}^{K}\right)$ optimal parameters obtained from $\left(\mathcal{P}^{N}\right)$ for observations $\tilde{z}^{K}$ and requires that $\bar{q}^{N}\left(\tilde{z}^{K}\right) \rightarrow \bar{q}\left(\tilde{z}^{0}\right)$ in some sense as $N \rightarrow \infty$ and $\tilde{z}^{K} \rightarrow \tilde{z}^{0}$ in $Z$, where $\bar{q}\left(\tilde{z}^{0}\right)$ is an optimal parameter for $(\mathcal{P})$ corresponding to observations $\tilde{z}^{0}$. These issues are carefully discussed in $[B],[B K]$ where it is shown that for both parameter convergence and method stability, it suffices to argue that

For arbitrary sequences $\left\{q^{N}\right\}$ in $Q$ converging to $q \in Q$, we have $\mathcal{C} u^{N}\left(t ; q^{N}\right) \rightarrow \mathcal{C} u(t ; q)$ as $N \rightarrow \infty$ for each $t \in(0, T)$.

Thus, certain fundamental aspects of approximation in inverse problems can be reduced to the convergence statement in (2.3) and we shall deal with conditions under which (2.3) can be guaranteed in the subsequent discussions of this paper.

In [BI], Banks and Ito developed a general framework for convergence and stability which we summarize here in an extended form to allow treatment of complex valued operators in complex state spaces (the sesquilinear forms introduced in [BI] were tacitly assumed to be generated by partial differential equations with real coefficients and the theory developed there was adequate for such examples). As in [BI], our goal is to isolate properties of the system (2.1) and the approximation families to insure that (2.3) holds. We state these properties in terms of conditions on a parameter dependent sesquilinear form arising from reformulating (2.1) in a weak or variational sense. To this end we consider the system as a variational equation holding for all $\phi \in V$ :

$$
\begin{aligned}
& (\dot{u}(t), \phi)+\sigma(q)(u(t), \phi)=\langle F(t, q), \phi>, \\
& u(0)=u_{0}(q) .
\end{aligned}
$$

Here $V$ and $H$ are complex Hilbert spaces with $V$ imbedded densely and continuously in the pivot space $H$; i.e. $V \hookrightarrow H=H^{*} \hookrightarrow V^{*}$. The sesquilinear form $\sigma(q): V \times V \rightarrow \mathrm{C}$ is related to the operator $A(q)$ as described below, $\langle$.$\rangle is the inner product in H,(\cdot, \cdot)$ is the duality product $(\cdot, \cdot)_{V^{*}, V}$ and equation $(2.4)$ is interpreted in the $V^{*}$ sense. The conditions on $\sigma$ needed are:

(A) Continuity in $q$ : For $q, \tilde{q} \in Q$, we have for all $\phi, \psi \in V$

$$
|\sigma(q)(\phi, \psi)-\sigma(\tilde{q})(\phi, \psi)| \leq d(q, \tilde{q})|\phi|_{V}|\psi|_{V}
$$

(B) V-Coercivity: There exists $c_{1}>0$ and some real $\lambda_{0}$ such that for $q \in Q, \phi \in V$, we have

$$
\operatorname{Re} \sigma(q)(\phi, \phi)+\lambda_{0}|\phi|_{H}^{2} \geq c_{1}|\phi|_{V}^{2} .
$$


(C) Boundedness in $q$ : There exists $c_{2}>0$ such that, for $q \in Q, \phi, \psi \in V$, we have

$$
|\sigma(q)(\phi, \psi)| \leq c_{2}|\phi|_{V}|\psi|_{V}
$$

Several remarks concerning these conditions are useful. First, we can weaken (A) slightly (see [BRR]): From (C) it follows that one can define $A(q)$ in $\mathcal{L}\left(V, V^{*}\right)$ by $\sigma(q)(\phi, \psi)=$ $(-A(q) \phi, \psi)_{V^{*}, V}$ and then one can replace condition (A) by the requirement that $q \rightarrow A(q)$ be continuous from $Q$ to $\mathcal{L}\left(V, V^{*}\right)$.

Moreover, for $\sigma: V \times V \rightarrow \mathrm{C}$ defined using real coefficients, i.e. real scalars, it suffices to replace (B) by the condition: $\sigma(q)(\phi, \phi)+\lambda_{0}|\hat{\phi}|_{H}^{2} \geq c_{1}|\phi|_{V}^{2}$ for all real valued functions $\phi$ in $V$ (this is condition (B) as stated in [BI]). To see this, we note that $\sigma(q)(\phi+i \psi, \phi+i \psi)$ $=\sigma(q)(\phi, \phi)+\sigma(q)(\psi, \psi)+i[\sigma(q)(\psi, \phi)-\sigma(q)(\phi, \psi)]$ and thus $\operatorname{Re} \sigma(q)(\phi+i \psi, \phi+i \psi)=$ $\sigma(q)(\phi, \phi)+\sigma(q)(\psi, \psi)$. Therefore, $\sigma(q)(\phi, \phi)+\lambda_{0}|\phi|_{H}^{2} \geq c_{1}|\phi|_{V}^{2}$ for all real valued $\phi$ in $V$ implies immediately that condition (B) above holds.

Under conditions (B) and (C), $\sigma$ defines operators $A(q)$ such that $\sigma(q)(\phi, \psi)=$ $\langle-A(q) \phi, \psi\rangle$ for $\phi \in \operatorname{dom}(A(q)), \psi \in V$ with $\operatorname{dom}(A(q))$ dense in $V$ (e.g., see [S]). Moreover, $A(q)$ is a sectorial operator with $(\lambda I-A(q)) \operatorname{dom}(A(q))=H$ for all $\lambda$ with $\operatorname{Re} \lambda \geq \lambda_{0}$; indeed $R_{\lambda}(A(q))=(\lambda I-A(q))^{-1}$ exists as a bounded operator on $H$ for all $\lambda$ in the complement of a sector with $\lambda_{0}$ as a vertex. The operator $A(q)$ generates an analytic semigroup $T(t ; q)$ which can be used in defining mild solutions of the system (2.4) or (2.1); i.e.,

$$
u(t ; q)=T(t ; q) u_{0}(q)+\int_{0}^{t} T(t-s ; q) F(s, q) d s .
$$

Galerkin approximations (e.g., systems (2.2)) for (2.5) can be developed in the context of sesquilinear forms satisfying conditions (B) and (C). Let $H^{N}$ be a family of finite dimensional subspaces of $H$ satisfying $H^{N} \subset V$ and the condition

$$
\begin{aligned}
& \text { For each } \phi \in V \text {, there exists } \hat{\phi}^{N} \in H^{N} \text { such that } \\
& \left|\phi-\hat{\phi}^{N}\right|_{V} \rightarrow 0 \text { as } N \rightarrow \infty
\end{aligned}
$$

(We note that many popular approximation schemes - e.g., linear splines and more generally, many finite element schemes - satisfy condition (C1).) To define $A^{N}(q): H^{N} \rightarrow H^{N}$, we restrict $\sigma(q)$ to $H^{N} \times H^{N}$ and denote this restriction by $\sigma^{N}(q)$. Then $\sigma^{N}$ satisfies conditions (A), (B), (C) on $H^{N} \times H^{N}$ which implies existence of a bounded linear operator $A^{N}(q)$ on $H^{N}$ satisfying $\sigma^{N}(q)(\phi, \psi)=<-A^{N}(q) \phi, \psi>$ for all $\phi, \psi \in H^{N}$. From (B) and (C) it follows that the $A^{N}(q)$ are uniformly dissipative (sectorial) and generate analytic semigroups $T^{N}(t ; q)$ on $H^{N}$. Solutions of the systems (2.2) are then given by

$$
u^{N}(t ; q)=T^{N}(t ; q) P^{N} u_{0}(q)+\int_{0}^{T} T^{N}(t-s ; q) P^{N} F(s, q) d s
$$


It can then be established that under conditions (A), (B), (C) and (C1), we have $u^{N}\left(t ; q^{N}\right) \rightarrow u(t ; q)$ in $V$ for arbitrary sequences $\left\{q^{N}\right\}$ with $q^{N} \rightarrow q$ in $Q$. Observe that this immediately yields (2.3) if the operator $\mathcal{C}$ possesses certain boundedness properties. The focus of this note involves cases where the operator $\mathcal{C}$ does not have such boundedness.

The arguments for the convergence $u^{N}\left(t ; q^{N}\right) \rightarrow u(t ; q)$ for the situation here (complex Hilbert spaces $V, H$ and complex valued sesquilinear form $\sigma$ ) are essentially the same as those in [BI]. Slight changes in the arguments for Theorem 2.2 of [BI] are necessary to treat the case of complex valued inner products and sesquilinear forms (essentially one need only use $\operatorname{Re} \sigma(\cdot, \cdot)$ and $\operatorname{Re}\langle\cdot, \cdot\rangle$ in some of the inequalities). In these arguments one does not use directly the analyticity properties of the semigroups $T(t ; q), T^{N}(t ; q)$. Rather one relies heavily on resolvent estimates of Tanabe [T] depending on the V-coercivity of $\sigma$ along with a resolvent convergence form of the Trotter-Kato approximation theorem of linear semigroup theory (see [BI] for details).

Our interest here is mainly in second order systems of the form

$$
\begin{aligned}
& \ddot{u}(t)+B(q) \dot{u}(t)+A(q) u(t)=f(t, q) \\
& u(0)=u_{0} \\
& \dot{u}(0)=v_{0}
\end{aligned}
$$

where $A(q)$ is a generalized stiffness operator and $B(q)$ is a generalized damping operator. Again we consider this equation in a weak or variational sense defined via parameter dependent sesquilinear forms in a complex Hilbert space $V \hookrightarrow H=H^{*} \hookrightarrow V^{*}$. We are given a stiffness sesquilinear form $\sigma_{1}(q): V \times V \rightarrow \mathrm{C}$ that is symmetric and satisfies the boundedness condition (C). Then there exists $A(q) \in \mathcal{L}\left(V, V^{*}\right)$ such that $\sigma_{1}(q)(\phi, \psi)=(A(q) \phi, \psi)_{V^{*}, V}$. We also have a damping sesquilinear form $\sigma_{2}(q): V \times V \rightarrow \mathrm{C}$ which satisfies (C) so that there exists $B(q) \in \mathcal{L}\left(V, V^{*}\right)$ with $\sigma_{2}(q)(\phi, \psi)=(B(q) \phi, \psi)_{V^{*}, V}$. We then reformulate the system (2.7) and seek solutions $u(t) \in V$ satisfying for all $\phi \in V$

$$
\begin{aligned}
& (\ddot{u}(t), \phi)+\sigma_{2}(q)(\dot{u}(t), \phi)+\sigma_{1}(q)(u(t), \phi)=\langle f(t, q), \phi\rangle \\
& u(0)=u_{0}, \dot{u}(0)=v_{0} .
\end{aligned}
$$

As is standard practice, we rewrite this in first order vector form on $\mathcal{H}=V \times H$ and $\mathcal{V}=V \times V$ in the coordinates $(u, \dot{u})$. To this end, define $\sigma(q): \mathcal{V} \times \mathcal{V} \rightarrow \mathrm{C}$ by

$$
\sigma(q)((u, v),(\phi, \psi))=-\langle v, \phi\rangle_{V}+\sigma_{1}(q)(u, \psi)+\sigma_{2}(q)(v, \psi)
$$

so that (2.8) may be rewritten as

$$
\begin{aligned}
& (\dot{w}(t), \chi)+\sigma(q)(w(t), \chi)=<F(t, q), \chi>_{H} \\
& w(0)=\left(u_{0}, v_{0}\right)
\end{aligned}
$$


for $w(t)=(u(t), \dot{u}(t))$ and $\chi=(\phi, \psi)$ in $\mathcal{V}$ with $F(t, q)=(0, f(t, q))$. Or, if as in the usual practice, we abuse notation and do not distinguish between row and column vectors, we may write this in equivalent operator form

$$
\begin{aligned}
\dot{w}(t) & =\mathcal{A}(q) w(t)+F(t, q) \\
w(0) & =\left(u_{0}, v_{0}\right)
\end{aligned}
$$

where $\sigma(q)(\chi, \xi)=(-\mathcal{A}(q) \chi, \xi)$ with

$$
\mathcal{A}(q)=\left[\begin{array}{cc}
0 & I \\
-A(q) & -B(q)
\end{array}\right] .
$$

For our treatment here (as for that in [BI]) we assume that $\sigma_{1}(q)$ satisfies conditions (A), (B), and (C). We further assume that $\sigma_{2}(q)$ satisfies conditions (A) and (C). Then the strength of the coercivity assumption on $\sigma_{2}(q)$ determines the properties of the semigroup generated by $\mathcal{A}(q)$. For example, if $\sigma_{2}(q)$ satisfies $(\mathrm{B})$, then $\mathcal{A}(q)$ is strongly $\mathcal{V}$-coercive and generates an analytic semigroup on $\mathcal{H}$. (Actually, $\mathcal{A}(q)$ is $\mathcal{V}_{q}$-coercive with $\mathcal{V}_{q}=V_{q} \times V_{q}$ where $V_{q}$ is $V$ taken with the equivalent inner product $\sigma_{1}(q)(\cdot, \cdot)-$ see [BI].) In [BI], it is assumed only that $\sigma_{2}$ is $H$-semicoercive: There exists $b \geq 0$ such that for all $\phi \in V$ we have Re $\sigma(q)(\phi, \phi) \geq b|\phi|_{H}^{2}$. In this case one can argue only that $\mathcal{A}(q)$ generates a strongly continuous semigroup on $\mathcal{H}$. The theory for second order systems with this weak damping is developed in [BI] in order to treat several forms of damping (spatial hysteresis, time hysteresis, bending rate damping) which are of physical interest and yet do not satisfy the strong $V$-coercivity assumption. The convergence theory obtained yields that $u^{N}\left(t ; q^{N}\right) \rightarrow u(t ; q)$ in $V$ norm, $\dot{u}^{N}\left(t ; q^{N}\right) \rightarrow \dot{u}(t ; q)$ in $H$ norm whenever $q^{N} \rightarrow q$.

As opposed to $[\mathrm{BI}]$, we wish to consider in this paper the case where $\sigma_{2}(q)$ does satisfy the strong $V$-coercivity condition (B) and hence $\mathcal{A}(q)$ is the infinitesimal generator of an analytic semigroup. We obtain immediately that $\sigma(q)$ of $(2.9)$ is $\mathcal{V}$-coercive and the first order theory outlined above can be applied directly to the system (2.10). This yields the convergence statement in $\mathcal{V}$ - i.e., $u^{N}\left(t ; q^{N}\right) \rightarrow u(t ; q)$ in $V, \dot{u}^{N}\left(t ; q^{N}\right) \rightarrow \dot{u}(t ; q)$ in $V$. A natural question arises as to whether we can use the analyticity of the semigroups in this case to obtain stronger results. The next section is devoted to results that yield an affirmative answer to this question.

\section{Analytic Semigroups and Approximation}

We first give a general approximation theorem for analytic semigroups that is a generalization of the well-known Trotter-Kato theorem [BK].

Theorem 3.1. Suppose we have complex Hilbert spaces $X$ and $X^{N}, N=1,2 \ldots$, with $X^{N} \subset X$. Let $P^{N}: X \rightarrow X^{N}$ denote the orthogonal projection of $X$ onto $X^{N}$ satisfying 
$P^{N} \rightarrow I$ strongly. Suppose that $A^{N}$ and $A$ are the infinitesimal generators of analytic semigroups $S^{N}(t)$ and $S(t)$ on $X^{N}$ and $X$ respectively that satisfy the following:

There exists a region $\Sigma=\sum_{\delta}=\left\{\lambda \in \mathbf{C}:\left|\arg \left(\lambda-\lambda_{0}\right)\right|<\frac{\pi}{2}+\delta\right\}$, where $\delta>0$, such that $\sum \cup\left\{\lambda_{0}\right\} \subset \rho(A) \cap_{N=1}^{\infty} \rho\left(A^{N}\right)$ and

(i) there exists a constant $M$ independent of $N$ such that

$$
\left|R_{\lambda}\left(A^{N}\right)\right| \leq \frac{M}{\left|\lambda-\lambda_{0}\right|}
$$

for all $\lambda \in \sum$ and $N=1,2, \ldots$;

(ii) for some $\lambda \in \sum$ and each $x \in X$ we have $R_{\lambda}\left(A^{N}\right) P^{N} x \rightarrow R_{\lambda}(A) x$.

Then we have

(iii) For each $x \in X, S^{N}(t) P^{N} x \rightarrow S(t) x$ uniformly in $t$ on compact subintervals of $[0, \infty)$;

(iv) For each $x \in X$ and integer $k \geq 1,\left(A^{N}\right)^{k} S^{N}(t) P^{N} x \rightarrow A^{k} S(t) x$ uniformly in $t$ on compact subintervals of $(0, \infty)$.

Proof. The statement of (iii) under the given conditions is just a variant of the well-known Trotter-Kato theorem and follows immediately from Theorem II.1.14 of [BK]. To argue result (iv), we first note the convergence in (ii) for some $\lambda \in \sum$ implies that the convergence holds for all $\lambda \in \Sigma$. In light of the resolvent bounds of (i), this follows from the identity for $\mu, \lambda \in \Sigma$

$$
R_{\mu}\left(A^{N}\right) P^{N}-R_{\mu}(A)=\left[I+(\lambda-\mu) R_{\mu}\left(A^{N}\right) P^{N}\right]\left[R_{\lambda}\left(A^{N}\right) P^{N}-R_{\lambda}(A)\right]\left[I+(\lambda-\mu) R_{\mu}(A)\right]
$$

which is readily established using the standard resolvent identity $R_{\lambda}(A)-R_{\mu}(A)=(\mu-$ ג) $R_{\lambda}(A) R_{\mu}(A)$.

The analyticity of the semigroups $S^{N}(t)$ and $S(t)$ allow us to write for $t>0$ (e.g., see [P])

$$
A^{k} S(t)=\frac{1}{2 \pi i} \int_{\Gamma} \lambda^{k} e^{\lambda t} R_{\lambda}(A) d \lambda
$$

and

$$
\left(A^{N}\right)^{k} S^{N}(t) P^{N}=\frac{1}{2 \pi i} \int_{\Gamma} \lambda^{k} e^{\lambda t} R_{\lambda}\left(A^{N}\right) P^{N} d \lambda .
$$

Here $\Gamma$ is a positively oriented contour through $\lambda_{0}$ lying in $\Sigma$ with $\arg \left(\lambda-\lambda_{0}\right)= \pm \nu$ for $\lambda \neq \lambda_{0}$ where $\nu$ is fixed in $\left(\frac{\pi}{2}+\delta-\varepsilon, \frac{\pi}{2}+\delta\right)$. The desired convergence results follow immediately from the inequality

$$
\begin{gathered}
\left|\left(A^{N}\right)^{k} S^{N}(t) \dot{P}^{N} x-A^{k} S(t) x\right| \\
\leq \frac{1}{2 \pi} \int_{\Gamma}|\lambda|^{k}\left|e^{\lambda t}\right| \quad\left|R_{\lambda}\left(A^{N}\right) P^{N} x-R_{\lambda}(A) x\right| d \lambda
\end{gathered}
$$


using the resolvent convergence of (ii) by noting that the integrand in this integral is dominated by an integrable function.

We now return to the second order systems of Section 2 - see (2.8), (2.9), (2.10) - and use Theorem 3.1 to obtain our main convergence results. As before we take $\mathcal{H}=V \times H$ and $\mathcal{V}=V \times V$. Let $\mathcal{H}^{N}=H^{N} \times H^{N}$ and $P^{N}$ be the orthogonal projection of $\mathcal{H}$ onto $\mathcal{H}^{N}$.

Theorem 3.2 Let $\sigma_{1}(q)$ and $\sigma_{2}(q)$ in (2.8) satisfy conditions $(A),(B)$ and $(C)$ and let $H^{N} \subset V$ satisfy condition (C1). Let $\left\{q^{N}\right\}$ be arbitrary in $Q$ with $q^{N} \rightarrow q$. Then we have

(i) The sesquilinear form $\sigma(q)$ given by (2.9) satisfies conditions $(A),(B),(C)$ in the norms of $\mathcal{V}$ and $\mathcal{H}$ and the operator $\mathcal{A}(q)$ defined via $\sigma(q)(\chi, \xi)=<-\mathcal{A}(q) \chi, \xi>_{\mathcal{H}}$ for $\chi \in \operatorname{dom}(\mathcal{A}(q))$ is the infinitesimal generator of an analytic semigroup $\mathcal{T}(t ; q)$ on $\mathcal{H}$.

(ii) Let $\mathcal{A}^{N}(q)$ denote the operator obtained by restricting $\sigma(q)$ to $\mathcal{H}^{N} \times \mathcal{H}^{N}$ and let $\mathcal{T}^{N}(t ; q)$ denote the corresponding analytic semigroups on $\mathcal{H}^{N}$. Then we have

(a) For each $\chi \in \mathcal{H}, \mathcal{T}^{N}\left(t ; q^{N}\right) P^{N} \chi \rightarrow \mathcal{T}(t ; q) \chi$ in $\mathcal{H}$ uniformly in $t$

on compact subintervals of $[0, \infty)$;

(b) For each $\chi \in \mathcal{H}$ and positive integer $k, \mathcal{A}^{N}\left(q^{N}\right)^{k} \mathcal{T}^{N}\left(t ; q^{N}\right) P^{N} \chi \rightarrow \mathcal{A}(q)^{k} \mathcal{T}(t ; q) \chi$

in $\mathcal{H}$ uniformly in $t$ on compact subintervals of $(0, \infty)$.

The proof of this theorem is now rather straightforward. We first note that it is readily shown with routine calculations that $\sigma_{1}, \sigma_{2}$ satisfying (A), (B), (C) in the $V$ and $H$ norms imply that $\sigma$ satisfies (A), (B), (C) with the norms of $\mathcal{V}=V \times V$ and $\mathcal{H}=V \times H$. Result (i) of the theorem thus is established.

For result (ii), we apply Theorem 3.1 with $X=\mathcal{H}=V \times H$ and $X^{N}=\mathcal{H}^{N}=H^{N} \times H^{N}$ along with arguments of [BI]. Restricting $\sigma\left(q^{N}\right)$ to $\mathcal{H}^{N} \times \mathcal{H}^{N}$ to obtain the operators $A^{N}=$ $\mathcal{A}^{N}\left(q^{N}\right)$, we have that condition (B) is satisfied in $\mathcal{H}^{N}$ with the constants uniform in $N$. i.e., the uniform sector condition and the uniform resolvent bounds in (i) of Theorem 3.1 are readily seen to hold. For the resolvent convergence of (ii) we refer to Theorem 2.2 of [BI], noting that we have all the hypotheses of that theorem holding here in the sense of the $\mathcal{V}$ and $\mathcal{H}$ norms. Hence the same arguments (modified slightly as mentioned in Section 2 above to account for complex Hilbert spaces $\mathcal{V}$ and $\mathcal{H}$ ) given for Theorem 2.2 of [BI] can be used here to establish (ii) of Theorem 3.1. The convergence statements (a) and (b) of Theorem 
3.2 then follow directly from (iii) and (iv) of Theorem 3.1.

We are now in a position to use the results of Theorem 3.2 to address the question of stronger convergence results for $u^{N}\left(t ; q^{N}\right)$ to $u(t ; q)$ raised in connection with the observation operator $\mathcal{C}$ in Section 2. We first consider the case of homogeneous systems (i.e., $f=0$ in (2.8)) in which case $w^{N}\left(t ; q^{N}\right)=\left(u^{N}\left(t ; q^{N}\right), \dot{u}^{N}\left(t ; q^{N}\right)\right)=\mathcal{T}^{N}\left(t ; q^{N}\right) P^{N} \chi$ and $w(t ; q)=$ $(u(t ; q), \dot{u}(t ; q))=\mathcal{T}(t ; q) \chi$. For $\chi \in \operatorname{dom}(\mathcal{A}(q))$ we have $\dot{w}^{N}(t)=\mathcal{A}^{N}\left(q^{N}\right) \mathcal{T}^{N}\left(t ; q^{N}\right) P^{N} \chi$, $\ddot{w}^{N}(t)=\mathcal{A}^{N}\left(q^{N}\right)^{2} \mathcal{T}^{N}\left(t ; q^{N}\right) P^{N} \chi$, etc., while $\dot{w}(t)=\mathcal{A}(q) \mathcal{T}(t ; q) \chi, \ddot{w}(t)=\mathcal{A}(q)^{2} \mathcal{T}(t ; q) \chi$, etc. Thus, from statement (b) of Theorem 3.2 we find $\ddot{w}^{N}\left(t ; q^{N}\right) \rightarrow \ddot{w}(t ; q)$ in $\mathcal{H}$, which yields $u_{t t}^{N}\left(t ; q^{N}\right) \rightarrow u_{t t}(t ; q)$ in the $V$ norm, uniformly in $t$ on compact subintervals of $(0, \infty)$. Indeed, for all $k=1,2 \ldots$, we obtain $\frac{\partial^{h}}{\partial t^{h}} u^{N}\left(t ; q^{N}\right) \rightarrow \frac{\partial^{h}}{\partial t^{h}} u(t ; q)$ in $V$.

Since, as we shall see in the next section, many interesting examples involve $V \subset H^{2}(\Omega)$ with $\Omega \subset R^{1}$ or $\Omega \subset R^{2}$, and since in this case $V$ imbeds continuously in $C(\Omega)$, the above results guarantee pointwise (in $t$ and $x$ ) convergence of $u^{N}\left(q^{N}\right)$ and all its time deviatives to $u(q)$ and its time deviatives, respectively. Thus, observation operators related to laser vibrometers $\left(u_{t}\right)$ and accelerometers $\left(u_{t t}\right)$ are included in the convergence and method stability theory for output least squares inverse problems.

Similar results are available for the nonhomogeneous equation (2.8) (or (2.10)) if one places appropriate regularity conditions on $f$. To obtain these, one considers the representation theorems (mild solutions)

$$
\begin{aligned}
& w^{N}\left(t ; q^{N}\right)=\mathcal{T}^{N}\left(t ; q^{N}\right) P^{N} w_{0}+\int_{0}^{t} \mathcal{T}^{N}\left(t-s ; q^{N}\right) P^{N} F(s, q) d s \\
& w(t ; q)=\mathcal{T}(t ; q) w_{0}+\int_{0}^{t} \mathcal{T}(t-s ; q) F(s, q) d s
\end{aligned}
$$

and uses regularity conditions on mild solutions in $\mathcal{H}=V \times H$. For example, if $f(\cdot, q)$ is in $C^{1}([0, T], H)$, one can differentiate once, if $f(\cdot, q)$ is in $C^{2}([0, T], H)$, one can differentiate twice, etc. (see $[\mathrm{P}]$ ). Indeed, since we have analytic semigroups, we can weaken the conditions on $f$ needed for this procedure by considering special regularity theorems for mild solutions of Cauchy initial value problems in the case of analyticity (e.g., see Chapter 4.3 of [P]). For example, it suffices to have $f(\cdot, q) \in L^{1}(0, T ; H)$ and locally Hölder continuous in order to differentiate $w^{N}$ and $w$ once in the above representations.

\section{Examples}

In this section we present briefly several examples to which the above theory can be readily applied. In some cases this sharpens the convergence and stability results currently available in the literature; in other cases it provides new results for the associated inverse 
problems.

Example 4.1. We consider a cantilevered Euler-Bernoulli beam with Kelvin-Voigt damping (i.e., stress proportional to a linear combination of the strain and the strain rate). The beam is assumed fixed at $x=0$ and free at $x=\ell$, with the transverse displacement at time $t$ and position $x$ given by $u(t, x)$. Typical observations consist of acceleration $u_{t t}\left(t, x_{j}\right)$ or velocity $u_{t}\left(t, x_{j}\right)$ at several locations $x_{j}$. Balance of forces and moments yield the following system (we assume the linear mass density $\rho$ is normalized to unity):

$$
\begin{gathered}
\frac{\partial^{2} u}{\partial t^{2}}+\frac{\partial^{2}}{\partial x^{2}}\left\{E I \frac{\partial^{2} u}{\partial x^{2}}+c_{D} I \frac{\partial^{3} u}{\partial x^{2} \partial t}\right\}=f(t, x) \quad 0<x<\ell \\
u(t, 0)=\frac{\partial u}{\partial x}(t, 0)=0, \\
{\left[E I \frac{\partial^{2} u}{\partial x^{2}}+c_{D} I \frac{\partial^{3} u}{\partial x^{2} \partial t}\right]_{x=\ell}=0,} \\
{\left[\frac{\partial}{\partial x}\left\{E I \frac{\partial^{2} u}{\partial x^{2}}+c_{D} I \frac{\partial^{3} u}{\partial x^{2} \partial t}\right\}\right]_{x=\ell}=0 .}
\end{gathered}
$$

The parameters to be estimated in typical examples (see [BWIC], [BFWIC], [BIn]) are the stiffness and damping coefficients, $E I$ and $c_{D} I$, respectively. For a parameter set we choose $Q$ compact in

$$
\tilde{Q}_{\nu}=\left\{q=\left(E I, c_{D} I\right): q \in L^{\infty}(0, \ell) \times L^{\infty}(0, \ell), E I(x) \geq \nu>0, c_{D} I(x) \geq \nu\right\}
$$

For the state spaces $H$ and $V$ we take $H=H^{0}(0, \ell), V=H_{L}^{2}(0, \ell) \equiv\left\{\phi \in H^{2}(0, \ell): \phi(0)=\right.$ $\phi^{\prime}(0)=0$. Then in writing the system (4.1) in the form (2.8) we take

$$
\begin{aligned}
& \sigma_{1}(q)(\phi, \psi)=<E I D^{2} \phi, D^{2} \psi>_{0} \\
& \sigma_{2}(q)(\phi, \psi)=<c_{D} I D^{2} \phi, D^{2} \psi>_{0}
\end{aligned}
$$

where $D^{2}=\frac{\partial^{2}}{\partial x^{2}}$ and $\left\langle, \cdot>_{0}\right.$ is the inner product in $H^{0}(0, \ell)=L^{2}(0, \ell)$. For real valued $\phi$ in $V$ we have at once

$$
\sigma_{i}(q)(\phi, \phi) \geq \nu\left|D^{2} \phi\right|_{0} \geq c_{1}|\phi|_{V}^{2}
$$

which, as we have noted, implies

$$
\operatorname{Re} \sigma_{i}(q)(\phi, \phi) \geq c_{1}|\phi|_{V}^{2}
$$

for complex valued $\phi$ in $V$. It is equally trivial to argue that conditions (A) and (C) of Section 2 hold for this $\sigma_{1}(q), \sigma_{2}(q)$. For Galerkin schemes satisfying condition (C1) we thus have Theorem 3.2 applicable, and hence parameter convergence and method stability hold 
for the least squares problems $(\mathcal{P})$ and $\left(\mathcal{P}^{N}\right)$ whenever one formulates these with pointwise observations of either velocity or acceleration.

Example 4.2 For this example we return to the so-called "RPL experiment" discussed in some detail in [BGRW]. The focus of our attention is a cantilevered Euler-Bernoulli beam with a flexible gas hose and thruster nozzle attached to the free end as depicted in Figure 2.2 of [BGRW]. The structure is modeled as a uniform cantilevered beam with Kelvin-Voigt internal damping and tip mass with a mass-spring-dashpot assembly attached at the tip. Along with the usual damped Euler-Bernoulli beam equation

$$
\rho \frac{\partial^{2} u}{\partial t^{2}}+E I \frac{\partial^{4} u}{\partial x^{4}}+c_{D} I \frac{\partial^{5} u}{\partial x^{4} \partial t}=0, \quad 0<x<\ell,
$$

we have the force balance equation at the tip

$$
\begin{aligned}
{\left[m_{T} \frac{\partial^{2} u}{\partial t^{2}}\right.} & \left.-c_{D} I \frac{\partial^{4} u}{\partial x^{3} \partial t}-E I \frac{\partial^{3} u}{\partial x^{3}}\right]_{x=\ell} \\
& =c_{H}\left(\dot{y}(t)-\frac{\partial u}{\partial t}(t, \ell)\right)+k_{H}(y(t)-u(t, \ell))+f(t)
\end{aligned}
$$

and the hose assembly state equation

$$
m_{H} \ddot{y}(t)+c_{H}\left(\dot{y}(t)-\frac{\partial u}{\partial t}(t, \ell)\right)+k_{H}(y(t)-u(t, \ell))=0 .
$$

Here $\rho, E I$, and $c_{D} I$ are the usual beam parameters whereas $m_{T}$ represents the tip mass, $m_{H}$ is the hose mass, $k_{H}$ is the hose stiffness (the "spring" or restoring force constant), $c_{H}$ is the hose damping coefficient and $f(t)$ represents an externally applied force at the tip (firing of the tip mounted thrusters). Boundary conditions for the coupled state equations (4.2) and (4.4) include the tip force balance equation (4.3), the tip moment balance equation (assuming that the hose assembly has negligible rotational inertia)

$$
\left[E I \frac{\partial^{2} u}{\partial x^{2}}+c_{D} I \frac{\partial^{3} u}{\partial x^{2} \partial t}\right]_{x=\ell}=0
$$

and the zero displacement, zero slope conditions at the fixed end $x=0$

$$
u(t, 0)=\frac{\partial u}{\partial x}(t, 0)=0 .
$$

The structure is assumed initially at rest so that initial conditions are given by

$$
\begin{gathered}
u(0, x)=\frac{\partial u}{\partial t}(0, x)=0 \\
y(0)=\dot{y}(0)=0 .
\end{gathered}
$$


The parameters to be estimated using accelerometer observations (see [BGRW]) include $q=\left(m_{T}, E I, c_{D} I, m_{H}, c_{H}, k_{H}\right)$, which is to be chosen from a compact subset $Q \subset R_{+}^{6}$.

To write the system (4.2)-(4.6) in weak or variational form, we use the state variable $\hat{u}(t)=(y(t), u(t, \ell), u(t, \cdot))$ in the state space $H=R^{2} \times H^{0}(0, \ell)$ with inner product

$$
<(\zeta, \eta, \phi),(\lambda, \mu, \psi)>_{H}=\zeta \lambda+\eta \mu+<\phi, \psi>_{0} .
$$

For the space $V$ we choose $V=\left\{(\zeta, \eta, \phi) \in H: \phi \in H^{2}(0, \ell), \phi(0)=D \phi(0)=0, \eta=\phi(\ell)\right\}$ with inner product

$$
<(\zeta, \phi(\ell), \phi),(\lambda, \psi(\ell), \psi)>_{V}=(\zeta-\phi(\ell))(\lambda-\psi(\ell))+\left\langle D^{2} \phi, D^{2} \psi>_{0} .\right.
$$

The stiffness and damping sesquilinear forms are given for $\hat{\phi}=(\zeta, \phi(\ell), \phi), \hat{\psi}=(\lambda, \psi(\ell), \psi)$ in $V$ by

$$
\begin{gathered}
\sigma_{1}(q)(\hat{\phi}, \hat{\psi})=k_{H}(\zeta-\phi(\ell))(\lambda-\psi(\ell))+E I<D^{2} \phi, D^{2} \psi>_{0} \\
\sigma_{2}(q)(\hat{\phi}, \hat{\psi})=c_{H}(\zeta-\phi(\ell))(\bar{\lambda}-\bar{\psi}(\ell))+c_{D} I<D^{2} \phi, D^{2} \psi>_{0} .
\end{gathered}
$$

We also need an operator $\mathcal{M}(q) \in \mathcal{L}(H)$ given by

$$
\mathcal{M}(q)(\zeta, \eta, \phi)=\left(m_{H} \zeta, m_{T} \eta, \rho \phi\right) .
$$

This operator can be extended to $\mathcal{L}\left(V^{*}\right)$ in an obvious manner. Then the system (4.2)-(4.6) can be written in variational form for the state $\hat{u}(t) \in V$ to satisfy for all $\hat{\phi} \in V$

$$
\begin{aligned}
& \left(\mathcal{M}(q) \hat{u}_{t t}(t), \hat{\phi}\right)+\sigma_{2}(q)\left(\hat{u}_{t}(t), \hat{\phi}\right)+\sigma_{1}(q)(\hat{u}(t), \hat{\phi})=\langle F(t), \hat{\phi}> \\
& \hat{u}(0)=\hat{u}_{t}(0)=0,
\end{aligned}
$$

where $F(t)=(0, f(t), 0)$. Since, for $m_{H}, m_{T}, \rho$ positive, the operator $\mathcal{M}(q)$ is invertible, equation (4.7) is obviously equivalent to an equation of the form (2.8). Thus the theory of Sections 2 and 3 is applicable if $\sigma_{1}$ and $\sigma_{2}$ satisfy the requisite hypotheses. If $Q$ is bounded below in $R_{+}^{6}$, it is readily seen that both $\sigma_{1}$ and $\sigma_{2}$ satisfy conditions (A), (B), and (C). For example, we see immediately for $\hat{\phi}=(\zeta, \phi(\ell), \phi) \in V$

$$
\begin{aligned}
\sigma_{2}(q)(\hat{\phi}, \hat{\phi}) & =c_{H}(\zeta-\phi(\ell))^{2}+c_{D} I\left|D^{2} \phi\right|_{0}^{2} \\
& \geq c_{1}\left\{(\zeta-\phi(\ell))^{2}+\left|D^{2} \phi\right|_{0}^{2}\right\}=c_{1}|\hat{\phi}|_{V}^{2} .
\end{aligned}
$$

Similar arguments hold for $\sigma_{1}(q)$.

Application of the theory in Sections 2 and 3 substantially sharpens the results given in [BGRW]. In that paper the main results (see Lemma 3.1) yield

$$
\int_{0}^{T}\left|\hat{u}_{t t}^{N}\left(t ; q^{N}\right)-\hat{u}_{t t}(t ; q)\right|_{H} d t \rightarrow 0
$$


as $N \rightarrow \infty$. The arguments are rather tedious and require the assumption $\hat{u}(q) \in H^{2}(0, T ; V)$ on the limit function. Note also that (4.8) would require continuous time acceleration observations be used in the least squares criterion.

In contrast, the theory of this paper yields (since $H^{2}$ embeds compactly in $\left.C\right) u^{N}\left(t, x ; q^{N}\right) \rightarrow$ $u(t, x ; q), \dot{u}^{N}\left(t, x ; q^{N}\right) \rightarrow \dot{u}(t, x ; q)$ uniformly in $x \in[0, \ell]$ for each $t \in[0, T]$ as well as $\hat{u}_{t t}^{N}\left(t ; q^{N}\right) \rightarrow \hat{u}_{t t}\left(t ; q^{N}\right)$ in $V$ for each $t$, which permits sampled time acceleration observations. The arguments are simple (given the theory developed above) and do not require the a priori smoothness assumption on $\hat{u}(q)$.

Example 4.3 As a final example, we briefly describe the models for two dimensional grid structures ("plates with holes") developed and investigated in $[R]$ and [BR]. We use LoveKirchoff plate theory with Kelvin-Voigt damping. The "plate" is rectangular, e.g., on $(x, y) \in$ $\left[0, \ell_{1}\right] \times\left[0, \ell_{2}\right]$, with rectangular holes periodically placed to produce a thin planar grid. It is assumed to be hanging vertically, clamped at the top, with the other three edges free. The basic equation for transverse displacements $w(t, x, y)$ is given by

$$
\rho h \frac{\partial^{2} w}{\partial t^{2}}+\frac{\partial^{2} M^{x}}{\partial x^{2}}+2 \frac{\partial^{2} M^{x y}}{\partial x \partial y}+\frac{\partial^{2} M^{y}}{\partial y^{2}}=f
$$

where $h$ is the thickness of the plate, the bending moments $M^{x}, M^{v}$ about the $x$ and $y$ axes, respectively, are given by

$$
\begin{aligned}
& M^{x}=\frac{E I}{1-\nu^{2}}\left\{\frac{\partial^{2} w}{\partial x^{2}}+\nu \frac{\partial^{2} w}{\partial y^{2}}\right\}+\frac{c_{D} I}{1-\nu^{2}}\left\{\frac{\partial^{3} w}{\partial x^{2} \partial t}+\nu \frac{\partial^{3} w}{\partial y^{2} \partial t}\right\} \\
& M^{y}=\frac{E I}{1-\nu^{2}}\left\{\frac{\partial^{2} w}{\partial y^{2}}+\nu \frac{\partial^{2} w}{\partial x^{2}}\right\}+\frac{c_{D} I}{1-\nu^{2}}\left\{\frac{\partial^{3} w}{\partial y^{2} \partial t}+\nu \frac{\partial^{3} w}{\partial x^{2} \partial t}\right\}
\end{aligned}
$$

and the twisting moment is given by

$$
M^{x y}=\frac{E I}{1-\nu^{2}}\left\{\frac{\partial^{2} w}{\partial x \partial y}-\nu \frac{\partial^{2} w}{\partial x \partial y}\right\}+\frac{c_{D} I}{1-\nu^{2}}\left\{\frac{\partial^{3} w}{\partial x \partial y \partial t}-\nu \frac{\partial^{3} w}{\partial x \partial y \partial t}\right\}
$$

Here $\nu$ is Poisson's ratio. For the boundary conditions at the top (clamped) we have the essential boundary conditions $w=\frac{\partial w}{\partial y}=0$ (the top corresponds to the $x$-axis). The plate is free on the other outer edges and on the edges of the holes, where the natural boundary conditions of zero moment and zero shear are required. For example, on a free edge parallel to the $y$-axis this results in the conditions

$$
M^{x}=0, \quad \frac{\partial M^{x}}{\partial x}+2 \frac{\partial M^{x y}}{\partial y}=0 .
$$

As shown in $[R],[B R]$, one can readily define the corresponding stiffness and damping sesquilinear forms $\sigma_{1}, \sigma_{2}$ on $V \times V$, where $V=\left\{\phi \in H^{2}(\Omega): \phi=\frac{\partial \phi}{\partial y}=0\right.$ along $\left.y=0\right\}, \Omega$ 
is $\left[0, \ell_{1}\right] \times\left[0, \ell_{2}\right]$ less the holes, and argue the needed $V$-coerciveness along with conditions (A) and (C). In this case, the state space is $H=H^{0}(\Omega)$. The theory of Sections 3 and 4 can thus be shown to hold for this example.

\section{References}

[B] Banks, H.T., On a variational approach to some parameter estimation problems, in "Distributed Parameter Systems," Springer L.N. in Control and Info. Sci., Vol. 75, 1985, pp. 1-23.

[BGRW] Banks, H.T., Gates, S.S., Rosen, I.G. and Wang, Y., The identification of a distributed parameter model for a flexible structure, SIAM J. Control Opt., $\underline{26}$, (1988), 743-762.

[BI] Banks, H.T. and Ito, K., A unified framework for approximation in inverse problems for distributed parameter systems, Control-Theory and Adv. Tech., $\underline{4}$, (1988), pp. $73-90$.

[BIn] Banks, H.T. and Inman, D.J., On damping mechanisms in beams, CAMS Rep.\# 89-3, University of Southern California, September 1989; ASME J. Applied Mech., submitted.

[BK] Banks, H.T. and Kunisch, K., "Estimation Techniques for Distributed Parameter Systems," Birkhäuser, Boston, 1989.

[BR] Banks, H.T. and Rebnord, D.A., Estimation of material parameters for grid structures, to appear.

[BRR] Banks, H.T., Reich, S. and Rosen, I.G., Estimation of nonlinear damping in second order distributed parameter systems, CAMS Rep.\#89-1, University of Southern California, September, 1989; Control-Theory and Adv. Tech., submitted.

[BFWIC] Banks, H.T., Fabiano, R.H., Wang, Y., Inman, D.J. and Cudney, H.H., Spatial versus time hysteresis in damping mechanisms, Proc. 27th IEEE Conf. Dec. Control (1988), pp. 1674-1677.

[BWIC] Banks, H.T., Wang, Y., Inman, D.J. and Cudney, H.H, Parameter identification techniques for the estimation of damping in flexible structures experiments, Proc. 26th IEEE Conf. Dec. Control (1987), pp. 1392-1395.

[P] Pazy, A., "Semigroups of Linear Operators and Applications to Partial Differential Equations," Springer-Verlag, New York, 1983. 
[R] Rebnord, D.A., Parameter estimation for two-dimensional grid structures, $\mathrm{PhD}$. Thesis, Brown University, May, 1989.

[S] Showalter, R.E., "Hilbert Space Methods for Partial Differential Equations," Pitman, London, 1979.

[T] Tanabe, H., "Equations of Evolution," Pitman, London, 1979. 


$$
-
$$$$
=
$$

$\equiv$
$\equiv$

$=$
$=$

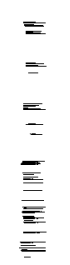

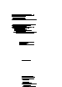




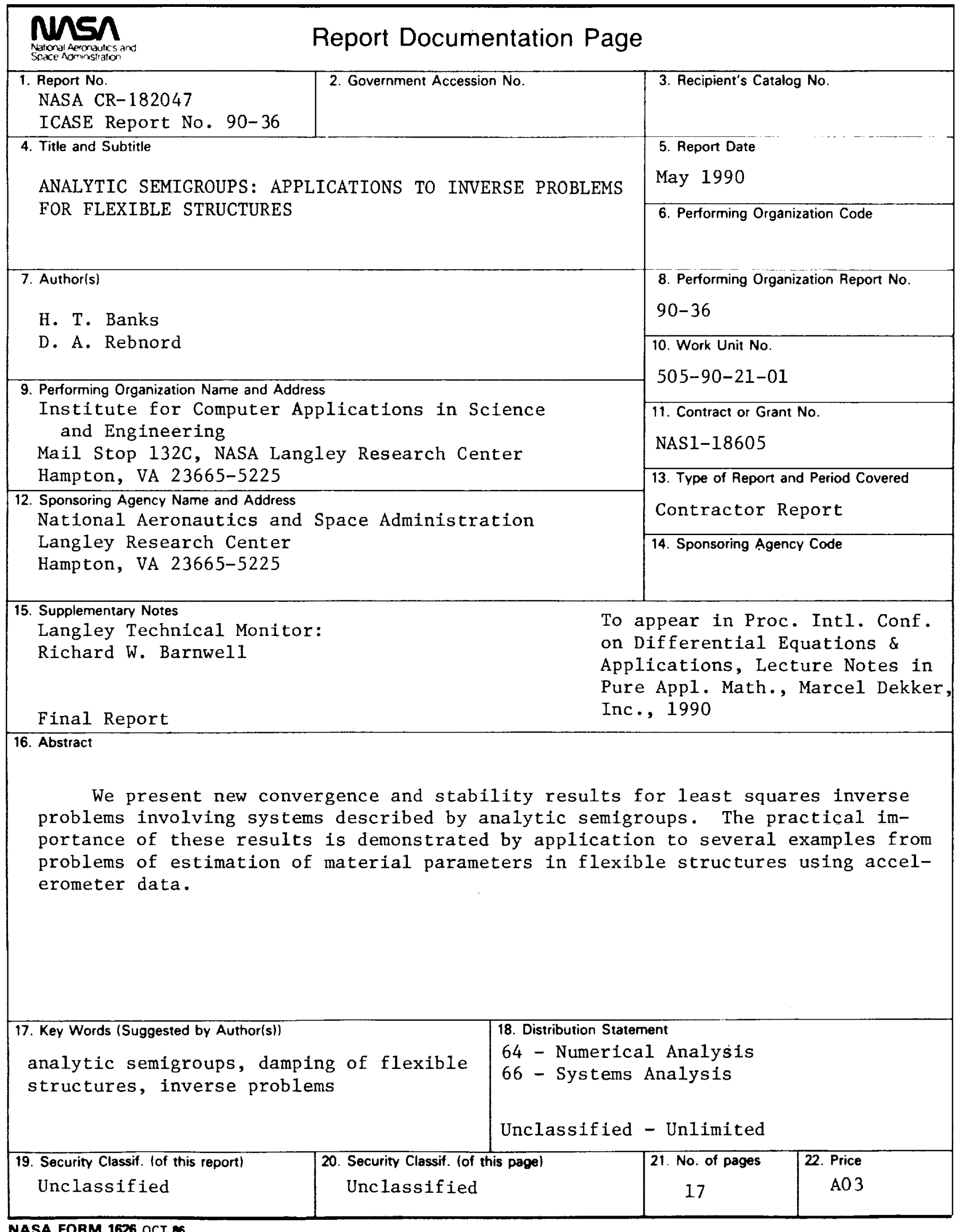


